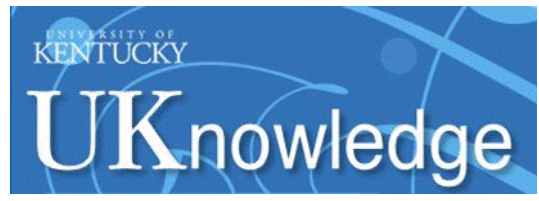

University of Kentucky

UKnowledge

9-1959

\title{
Coefficient Problems for Functions Regular in an Ellipse
}

Wimberly C. Royster

University of Kentucky

Follow this and additional works at: https://uknowledge.uky.edu/math_facpub

Part of the Mathematics Commons

Right click to open a feedback form in a new tab to let us know how this document benefits you.

\section{Repository Citation}

Royster, Wimberly C., "Coefficient Problems for Functions Regular in an Ellipse" (1959). Mathematics Faculty Publications. 3.

https://uknowledge.uky.edu/math_facpub/3

This Article is brought to you for free and open access by the Mathematics at UKnowledge. It has been accepted for inclusion in Mathematics Faculty Publications by an authorized administrator of UKnowledge. For more information, please contact UKnowledge@lsv.uky.edu. 


\section{Coefficient Problems for Functions Regular in an Ellipse}

Digital Object Identifier (DOI)

http://dx.doi.org/10.1215/S0012-7094-59-02634-1

\section{Notes/Citation Information}

Published in Duke Mathematical Journal, v. 26, no. 3, p. 361-371.

This article is republished by permission of the copyright holder, Duke University Press. Reuse of material, except as citation, is subject to Duke University Press's permission. 


\title{
COEFFICIENT PROBLEMS FOR FUNCTIONS REGULAR IN AN ELLIPSE
}

\author{
By W. C. Royster
}

1. Introduction. Let $f(z)$ be an analytic function which is regular in an ellipse with foci at \pm 1 . It is known that such a function possesses an expansion in a series of Tchebychef polynomials in the largest ellipse in which $f(z)$ is regular [8]. We shall be concerned in this paper with the problem of obtaining bounds on the coefficients in the expansion whenever $f(z)$ belongs to certain classes of functions which have specified mapping properties. The classes which we shall consider are

(1) typically real functions $(T)$

(2) functions that are univalent and convex in the direction of the imaginary $\operatorname{axis}(F)$

(3) functions starlike in the direction of the real axis $(R)$

(4) starlike functions $(S)$

(5) functions having a diametral line $(D)$.

The class of typically real functions was first studied for Taylor series by Rogosinski [6] and later for Laurent series by Nehari and Schwarz [3]. The classes $F$ and $R$ were studied for Taylor series by Robertson [4], [5]. The class $S$ has been studied for Taylor series by many authors and for Laurent series by Nehari and Schwarz [3], while the class $D$ was studied by Umezawa [7] and De Bruijn [2].

2. The class $T$. The function $f(z)$ is said to be typically real in $E$ if it is real when and only when $z$ is real. The assumption that $f(z) \varepsilon T$ is equivalent to the assumption that $\operatorname{Im} f(z)$. $\operatorname{Im} z$ possesses the same sign for any $z \varepsilon E$ for which $\operatorname{Im} z \neq 0$. Without loss of generality suppose $\operatorname{Im} f(z) \cdot \operatorname{Im} z>0$. Since $f(z)$ is regular in $E$, it has an expansion in a series of Tchebychef polynomials

$$
f(z)=\sum_{n=0}^{\infty} a_{n} T_{n}(z), \quad z \varepsilon E
$$

where $T_{n}(z)=\cos n\left(\cos ^{-1} z\right)$, which converges uniformly in $E$ [8].

First let us relate the class of typically real functions in $E$ to functions having positive real part in $E$. Let the parametric representation of $E$ be given by $z=a_{0} \cos t+i b_{0} \sin t, 0 \leq t<2 \pi$. Since $a_{0}^{2}-b_{0}^{2}=1$, we let $a_{0}=\cosh s_{0}$, $b_{0}=\sinh s_{0}, s_{0}>0$. Any ellipse which is confocal with $E$ and interior to $E$ can be represented by $z=a \cos t+i b \sin t, 0 \leq t<2 \pi$, with $a=\cosh s$, $b=\sinh s, 0<s \leq s_{0}$. Thus we may write $z=\cos (t-i s)$.

Received May 5, 1958. 
Theorem 2.1. If $f(z)$ is typically real in $E$, then

$$
\operatorname{Re}\left\{\left(b z-i a\left(1-z^{2}\right)^{\frac{1}{2}}\right) f(z)\right\} \geq 0 .
$$

Proof. $\operatorname{Im} f(z) \cdot \operatorname{Im} z>0$ is equivalent to $\sin t \operatorname{Im} f(z)>0$. Since $z=\cos$ $(t-i s)$ we have $\left(1-z^{2}\right)^{\frac{1}{2}}=\sin (t-i s)$, where the value of the radical is determined by continuity from the condition that at $t=0, z=a$ and $\left(1-z^{2}\right)^{\frac{1}{2}}=$ - ib. A simple calculation shows that $-i \sin t=b z-i a\left(1-z^{2}\right)^{\frac{1}{2}}$. Since $\sin t \operatorname{Im} f(z)=\operatorname{Re}\{-i \sin t f(z)\}$ the proof is complete.

TheOREM 2.2. Let $f(z)=\sum_{n=1}^{\infty} a_{n} T_{n}(z), a_{0}=0$, be regular and typically real in $E$. Then

$$
\left|a_{n}\right| \leq n\left|a_{1}\right|\left(\frac{R-R^{-1}}{R^{n}-R^{-n}}\right), \quad n=1,2, \cdots
$$

where $R=a+b$. The inequality is sharp.

Proof. Consider $G(z)=\left(b z-i a\left(1-z^{2}\right)^{\frac{1}{2}}\right) f(z)$. Then

$$
G(z)=-i \sin t \sum_{n=1}^{\infty} a_{n} \cos n(t-i s)
$$

$$
\begin{aligned}
& \operatorname{Re}\{G(z)\}=\sum_{n=1}^{\infty} a_{n} \sinh n s \sin n t \sin t \\
& =\frac{1}{2}\left\{a_{1} \sinh s+\sum_{n=1}^{\infty}\left[a_{n+1} \sinh (n+1) s-a_{n-1} \sinh (n-1) s\right] \cos n t\right\} .
\end{aligned}
$$

Since $\operatorname{Re}\{G(z)\} \geq 0$, a well known classical inequality for non-negative trigonometric series, yields

$$
\left|a_{n+1} \sinh (n+1) s-a_{n-1} \sinh (n-1) s\right| \leq 2\left|a_{1}\right| \sinh s .
$$

Using induction and the fact that $a_{0}=0$ we see that

$$
\left|a_{n}\right| \leq n\left|a_{1}\right| \frac{\sinh s}{\sinh n s}=n\left|a_{1}\right|\left(\frac{R-R^{-1}}{R^{n}-R^{-n}}\right) \text {. }
$$

In showing that (2.2) is sharp let us assume that $a_{1}=1$ in order to simplify the manipulations. From $[9 ; 535]$ we obtain the expansion of the Jacobian elliptic function $1 / s n^{2} u$ which, except for an additive real constant, is

$$
\frac{1}{\operatorname{sn}^{2}\left(\frac{2 K u}{\pi}\right)}=\left(\frac{\pi}{2 K}\right)^{2}\left[\csc ^{2} u-8 \sum_{n=1}^{\infty} \frac{n q^{2 n}}{1-q^{2 n}} \cos 2 n u\right] \text {, }
$$

valid for $|\operatorname{Im} u|<\log 1 / q$, where for our purpose $K=\pi / 2$ and $q=R^{-1}$. Let $u=\frac{1}{2}\left(\cos ^{-1} z+i \log R\right)$, then a simple computation yields 


$$
\begin{aligned}
\frac{1}{s n^{2}(u)} & =-\frac{8 q}{1-q^{2}} \sum_{n=1}^{\infty} n\left(\frac{R-R^{-1}}{R^{n}-R^{-n}}\right) \cos n\left(\cos ^{-1} z\right) \\
& =-\frac{8}{R-R^{-1}} \sum_{n=1}^{\infty} n\left(\frac{R-R^{-1}}{R^{n}-R^{n}}\right) T_{n}(z) \\
& =-\frac{8}{R-R^{-1}} f_{0}(z) .
\end{aligned}
$$

Hence, except for a real additive constant, the extremal function is

$$
f_{0}(z)=-\frac{1}{8} \cdot \frac{R-R^{-1}}{\operatorname{sn}^{2} \frac{1}{2}\left(\cos ^{-1} z+i \log R\right)} .
$$

To show $f_{0}(z)$ is typically real in $E$ we note, except for a positive multiplicative constant, that (see $[1 ; 38]$ )

$\operatorname{Im}\left\{f_{0}(z)\right\}=(2 s n t / 2 \mathrm{cnt} t / 2 d n t / 2)$

$$
\cdot s n[(\log R)-s] c n[(\log R)-s] d n[(\log R)-s] .
$$

However $e^{s} \leq e^{s o}=R$, hence $s \leq \log R$ and

$$
\begin{array}{ll}
\operatorname{Im} f_{0}(z) \geq 0, & 0 \leq t \leq \pi \\
\operatorname{Im} f_{0}(z) \leq 0, & \pi \leq t \leq 2 \pi
\end{array}
$$

which shows that $f_{0}(z) \varepsilon T$.

3. Functions convex in one direction. Suppose that $f(z)=\sum_{n=0}^{\infty} a_{n} T_{n}(z)$, $a_{n}$ real, is regular and convex in the direction of the imaginary axis whenever $z$ is in $E$. By convex in the direction of the imaginary axis we shall mean that the ellipse $z=\cos (t-i s), s \leq s_{0}$, is mapped by $f(z)$ onto a contour which is cut by a straight line parallel to the imaginary axis in at most two points.

Since the coefficients are real and $T_{n}^{*}(z)=T_{n}\left(z^{*}\right)\left(z^{*}\right.$ denotes the complex conjugate of $z$ ), the contour is symmetrical about the real axis. Let $f(z)=$ $U(s, t)+i V(s, t)$. If $f(z) \varepsilon F$, then Re $\{f(z)\}$ is a monotonically decreasing function of $t$ for $0<t<\pi$ and a monotonically increasing function of $t$ for $\pi<t<2 \pi$. We now have the following

Lemma 3.1. If $f(z)=\sum_{n=0}^{\infty} a_{n} T_{n}(z), a_{n}$ real, belongs to $F$ for $z$ in $E$, then $\left(z^{2}-1\right)^{\frac{1}{2}} f^{\prime}(z)$ is typically real, and conversely.

Proof.

$$
\frac{d f}{d t}=\frac{d f}{d z} \frac{\partial z}{\partial t}, \quad z=\cos (t-i s)
$$

so that

$$
\frac{\partial U}{\partial t}+i \frac{\partial V}{\partial t}=-\left(1-z^{2}\right)^{\frac{1}{2}} f^{\prime}(z)
$$


Hence $\operatorname{Im}\left\{\left(z^{2}-1\right)^{\frac{1}{2}} f^{\prime}(z)\right\}=\partial U / \partial t$. Using the known properties of $\partial U / \partial t$ we have that $\left(z^{2}-1\right)^{\frac{1}{2}} f^{\prime}(z)$ is typically real in $E$.

THEOREM 3.2. Let $f(z)=\sum_{n=1}^{\infty} a_{n} T_{n}(z), a_{0}=0, a_{n}$ real, be regular and convex in the direction of the imaginary axis for $z$ in $E$, then

$$
\left|a_{n}\right| \leq\left|a_{1}\right|\left(\frac{R+R^{-1}}{R^{n}+R^{-n}}\right), \quad n=1,2, \cdots .
$$

Proof. Let $G(z)=\left(b z-i a\left(1-z^{2}\right)^{\frac{1}{2}}\right)\left(z^{2}-1\right)^{\frac{1}{2}} f^{\prime}(z)$. By Lemma 3.1 and Theorem 2.1 we have that $G(z)$ has positive real part in $E$. Now

$$
f^{\prime}(z)=\sum_{n=1}^{\infty} n a_{n}\left(1-z^{2}\right)^{-\frac{1}{2}} \sin n\left(\cos ^{-1} z\right),
$$

and

$$
\left(z^{2}-1\right)^{\frac{1}{2}} f^{\prime}(z)=i \sum_{n=1}^{\infty} n a_{n} \sin n(t-i s) .
$$

Therefore

$$
\begin{aligned}
\operatorname{Re}\{G(z)\} & =\operatorname{Re}\left\{-i \sin t\left(z^{2}-1\right)^{\frac{1}{2}} f^{\prime}(z)\right\} \\
& =\sum_{n=1}^{\infty} n a_{n} \sin t \sin n t \cosh n s . \\
& =A_{1}+\sum_{n=1}^{\infty}\left(A_{n+1}-A_{n-1}\right) \cos n t
\end{aligned}
$$

where $A_{n}=n a_{n} \cosh n s$.

Since $\operatorname{Re}\{G(z)\} \geq 0$ we have by the aforementioned property of positive trigonometric series that

$$
\left|A_{n+1}-A_{n-1}\right| \leq 2\left|A_{1}\right|
$$

and since $A_{0}=0$, that $\left|A_{n}\right| \leq n\left|A_{1}\right|$ which establishes (3.2).

In order to investigate the sharpness of (3.2) let us consider the function $F_{0}^{\prime}(u)=i$ cn $u /$ sn $u-1$ and let $u=\frac{1}{2}\left(\cos ^{-1} z+i \log R\right)$. Then according to $[10 ; 512]$

$$
F_{0}(u)=-1+i\left[\cot u-4 \sum_{n=1}^{\infty} \frac{q^{2 n}}{1+q^{2 n}} \sin 2 n u\right]
$$

valid for $|\operatorname{Im} u|<\log R$ where $R=q^{-1}$. A short computation shows that

$$
F_{0}(z)=4 \sum_{n=1}^{\infty} \frac{\cos n\left(\cos ^{-1} z\right)}{R^{n}+R^{-n}} \text {. }
$$

Hence

$$
f_{0}(z)=\frac{R+R^{-1}}{4} F_{0}(z)
$$


yields equality in (3.2). By Lemma $3.1 f_{0}(z)$ will be convex in the direction of the imaginary axis if $\left(z^{2}-1\right)^{\frac{1}{2}} f_{0}^{\prime}(z)$ is typically real in $E$. However, $f_{0}^{\prime}(z)$ is given by

$$
\left(z^{2}-1\right)^{\frac{1}{2}} f_{0}^{\prime}(z)=\frac{d n \frac{1}{2}\left(\cos ^{-1} z+i \log R\right)}{\operatorname{sn}^{2} \frac{1}{2}\left(\cos ^{-1} z+i \log R\right)} .
$$

Using the fact that $z=\cos (t-i s)$ and separating into real and imaginary parts (see [1]) we find

$$
\begin{array}{rl}
\operatorname{Im}\left\{f_{0}^{\prime}(z)\right\}=M s n t / 2 c n t / 2 & s n(\log R-s)\left\{k^{2} s n^{2} t / 2 d n^{2}(\log R-s)\right. \\
& +d n^{2} t / 2 c n^{2}(\log R-s)\left[2 c n^{2}(\log R-s)\right. \\
& \left.\left.+k^{2} s n^{2}(\log R-s)\left(2-c n^{2} t / 2\right)\right]\right\}
\end{array}
$$

where $M$ is a positive constant and $0 \leq t \leq 2 \pi$. (3.10) shows that $\left(z^{2}-1\right)^{\frac{1}{2}}$ $f_{0}^{\prime}(z)$ is typically real since the quantity in the braces in the right member of (3.10) is positive.

4. Functions starlike in the direction of the real axis. Let $W=f(z)=$ $\sum_{n=1}^{\infty} a_{n} T_{n}(z)$, be regular and single-valued in $E$. Suppose $f(z)$ is starlike in the direction of the real axis, i.e. suppose for each $s, 0<s \leq s_{0}, f(z)$ maps the ellipse $z=\cos (t-i s)$ onto a curve which is cut by the real axis in exactly two points, or $\operatorname{Im}\{f(\cos (t-i s))\}$ changes sign twice.

Lemma 4.1. Let $f(z)=\sum_{n=1}^{\infty} a_{n} T_{n}(z)$ be regular in $E$ and $\operatorname{Im}\{f(z)\}$ change sign exactly twice, say at $t=t_{1}, t_{2}$. There exist real numbers $\mu$ and $\nu$ such that if

$$
g(z)=\left(a z-i b\left(1-z^{2}\right)^{\frac{1}{2}}-\cos \nu\right) f(\cos ((t+\mu)-i s)),
$$

then $\operatorname{Im} g(z)$ does not change sign for $z$ on $E$.

Proof. Since $a z-i b\left(1-z^{2}\right)^{\frac{1}{2}}=\cos t$ we have

$$
\operatorname{Im}\{g(z)\}=(\cos t-\cos \nu) \operatorname{Im}\{f(\cos ((t+\mu)-i s))\} .
$$

Take $\mu=\left(t_{1}+t_{2}\right) / 2$ and $\nu=\left(t_{2}-t_{1}\right) / 2$. Now $\operatorname{Im}\{f(\cos (t+\mu)-i s)\}$ changes sign at $t+\mu=t_{1}, t_{2}$, or $t= \pm\left(t_{2}-t_{1}\right) / 2= \pm \nu$. But $\cos t-\cos \nu$ changes sign at $t= \pm \nu,-\pi \leq t \leq \pi$, hence $\operatorname{Im}\{g(z)\}$ does not change sign at $t= \pm \nu$. Consequently $\operatorname{Re}\{i g(z)\}$ or $\operatorname{Re}\{-i g(z)\}$ is positive for $z$ on $E$.

Theorem 4.2. Let $f(z)=\sum_{n=1}^{\infty} a_{n} T_{n}(z)$ be regular and single-valued in $E$. Further, let $\operatorname{Im}\{f(z)\}$ change sign exactly twice on $E$. Then

$$
\left|a_{n} R^{n}-a_{n}^{*} R_{n}^{-n}\right| \leq n^{2}\left|a_{1} R-a_{1}^{*} R^{-1}\right| .
$$

Proof. By Lemma (4.1) there exist $\mu$ and $\nu$ such that

$$
g(z)=\left[a z-i b\left(1-z^{2}\right)^{\frac{1}{2}}-\cos \nu\right] f(\cos ((t+\mu)-i s))
$$


is regular on $E$ and $\operatorname{Im}\{g(z)\}$ does not change signs on $E$. Since

$$
g(z)=(\cos t-\cos \nu) \sum_{n=1}^{\infty} a_{n} T_{n}(\cos ((t+\mu)-i s)),
$$

with $a_{n}=\alpha_{n}+\beta_{n}$, then

$$
\begin{aligned}
& \operatorname{Re}\{-i g(z)\} \\
& =(\cos t-\cos \nu) \sum_{n=1}^{\infty}\left(\alpha_{n} \sinh n s \sin n \mu+\beta_{n} \cosh n s \cos n \mu\right) \cos n t \\
& \quad+\left(\alpha_{n} \sinh n s \cos n \mu-\beta_{n} \cosh n s \sin n \mu\right) \sin n t
\end{aligned}
$$

where

$$
\begin{aligned}
& A_{n}=\alpha_{n} \sinh n s \sin n \mu+\beta_{n} \cosh n s \cos n \mu \\
& B_{n}=\alpha_{n} \sinh n s \cos n \mu-\beta_{n} \cosh n s \sin n \mu, \quad n=1,2, \cdots \\
& A_{0}=B_{0}=0 .
\end{aligned}
$$

Hence

$$
\begin{aligned}
& \operatorname{Re}\{-i g(z)\}=\frac{1}{2} A_{1}+\frac{1}{2} \sum_{n=1}^{\infty} {\left[A_{n+1}+A_{n-1}-2 A_{n} \cos \nu\right] \cos n t } \\
&+\left[B_{n+1}+B_{n-1}-2 B_{n} \cos \nu\right] \sin n t \geq 0 .
\end{aligned}
$$
that

It follows by the aforementioned property of positive trigonometric series

We note that $A_{n}-i B_{n}=-i e^{i n \mu} / 2\left(a_{n} R^{n}-a_{n}^{*} R^{-n}\right)$ and $A_{1}=\operatorname{Re}\{-i / 2$ $\left.\left(a_{1} R-a_{1}^{*} R^{-1}\right) e^{i \mu}\right\}$ so that (4.8) becomes

$$
\begin{aligned}
\mid\left(a_{n+1} R^{n+1}-a_{n+1}^{*} R^{-(n+1)}\right) e^{i(n+1) \mu}+\left(a_{n-1} R^{n-1}-a_{n-1}^{*} R^{-(n-1)}\right) e^{i(n-1) \mu} \\
-2\left(a_{n} R^{n}-a_{n}^{*} R^{-n}\right) e^{i n \mu} \cos \nu|\leq 2| a_{1} R-a_{1}^{*} R^{-1} \mid .
\end{aligned}
$$

Let $c_{n}=\left(a_{n} R^{n}-a_{n} R^{-n}\right) e^{i n \mu} ;$ then the left hand member of (4.9) may be written as

$$
c_{n+1}-2 c_{n} \cos \nu+c_{n-1}=b_{n}, \quad n=1,2, \cdots, \text { with } b_{0}=c_{1}
$$

which by (4.9) gives $\left|b_{n}\right| \leq 2\left|b_{0}\right|$. One can show from (4.10) that

$$
c_{n}=\sum_{p=0}^{n-1} b_{p} \frac{\sin (n-p) \nu}{\sin \nu}
$$


Substituting for $b_{0}$ we have

$$
c_{n}=c_{1} \frac{\sin n \nu}{\sin \nu}+\sum_{p=1}^{n-1} b_{p} \frac{\sin (n-p) \nu}{\sin \nu}
$$

and

$$
\begin{aligned}
\left|c_{n}\right| \leq n\left|c_{1}\right|+\sum_{p=1}^{n+1}(n & -p)\left|b_{p}\right| \\
& \leq n\left|c_{1}\right|+2\left|c_{1}\right| \sum_{p=1}^{n-1}(n-p)=n^{2}\left|c_{1}\right|
\end{aligned}
$$

Hence

$$
\left|a_{n} R^{n}-a_{n}^{*} R^{-1}\right| \leq n^{2}\left|a_{1} R-a_{1}^{*} R^{-1}\right| .
$$

It has been tacitly assumed that $f(z)$ is regular on $E$, however it is sufficient to assume that $f(z)=f(\cos (t-i s))$ is regular in and on the ellipse $E_{1}$ given by $z=\cos \left(t-i s_{1}\right), s_{1}<s_{0}$. Upon proving the result for $f(z)$ regular in the closure of $E_{1}$ the corresponding results follow for $f(z)$ regular in $E$ by letting $s_{1} \rightarrow s_{0}$.

Inequality (4.14) has greater geometric significance than is apparent in its present form. Upon separating into real and imaginary parts and squaring, we have

$$
\alpha_{n}^{2}\left(R^{n}-R^{-n}\right)^{2}+\beta_{n}^{2}\left(R^{n}+R^{-n}\right)^{2} \leq n^{4} \lambda^{2},
$$

where $\lambda^{2}=\alpha_{1}^{2}\left(R-R^{-1}\right)^{2}+\beta_{1}^{2}\left(R+R^{-1}\right)^{2}$. Inequality (4.15) implies that the coefficients in the expansion of $f(z)$ in Theorem 4.2 lie in the closed ellipse with center at the origin and semi-axes $n^{2} \lambda /\left(R^{n}-R^{-n}\right)$ and $n^{2} \lambda /\left(R^{n}+R^{-n}\right)$.

To study the question of sharpness of (4.15) let us consider

$$
F(u)=\frac{1}{16} \frac{d}{d u}\left(\frac{1}{s^{2}(u+i \log R+\pi / 4)}-\frac{1}{s n^{2}(u-\pi / 4)}\right)
$$

where $1 / s n^{2} u$ is given by (2.7). With $K=\pi / 2, K^{\prime}=\log R$ we have $q=R^{-2}$ and

$$
\frac{d}{d u}\left(\frac{1}{s n^{2} u}\right)=-8 i \sum_{n=1}^{\infty} \frac{n^{2}}{R^{2 n}-R^{-2 n}}\left(R^{2 n} e^{2 i n u}-R^{-2 n} e^{-2 i n u}\right)
$$

so that

$$
F(u)=\frac{1}{2} \sum_{n=1}^{\infty} \frac{i n^{2}}{R^{2 n}-R^{-2 n}}\left(R^{n} e^{-i n \pi / 2}-R^{-n} e^{i n \pi / 2}\right)\left(R^{n} e^{2 i n u}+R^{-n} e^{-2 i n u}\right) .
$$

Further, let $u=\frac{1}{2}\left(\cos ^{-1} z+i \log R\right)$; then $T_{n}(z)=\left(R^{n} e^{2 i n u} R^{-n} e^{-2 i n u}\right) / 2$ which yields

$$
F(u)=F_{1}(z)=\sum_{n=1}^{\infty} \frac{i n^{2}}{R^{2 n}-R^{-2 n}}\left(R^{n} e^{-i n \pi / 2}-R^{-n} e^{i n \pi / 2}\right) T_{n}(z) .
$$


It is easy to verify that the coefficients in (4.19) yield equality in (4.15), and (4.9) with $\nu=0$. From reduction formulae [1] it follows that

$$
F(u)=-\frac{1}{8} \operatorname{cn}(u-\pi / 4)\left(\frac{k^{\prime 2} k^{2} s n(u-\pi / 4)}{d n^{3}(u-\pi / 4)}-\frac{d n(u-\pi / 4)}{s n^{3}(u-\pi / 4)}\right)
$$

where $k$ is a parameter called the modulus associated with these elliptic functions and $k^{\prime}=\left(1-k^{2}\right)^{\frac{1}{2}}$ is the complementary modulus.

We shall exhibit the existence of an elliptic annulus which we shall call a boundary strip $G$, formed by $E$ and a confocal ellipse lying inside $E$, such that on any ellipse $E_{1}$ confocal with $E$ and with boundary in $G \cdot \operatorname{Im}\left\{F_{1}(z)\right\}$ changes sign exactly twice, provided $k^{\prime}>2^{-\frac{1}{2}}$. Let $E_{1}$ be given parametrically by $z=\cos$ $\left(t-i s_{1}\right)$, where $s_{1}=\log R_{1}, R_{1}<R_{0}$, so that on $E_{1}, u=\frac{1}{2}\left(t+i \log R_{0} / R_{1}\right)=$ $\frac{1}{2} t+i \epsilon, \epsilon=\frac{1}{2} \log R_{0} / R_{1}$. We shall first show that $\operatorname{Im}\left\{F_{1}(z)\right\}$ changes sign at least twice on $E_{1}$. We shall then show that $d / d t\left(\operatorname{Im}\left\{F_{1}(z)\right\}\right)$ vanishes exactly twice on $E_{1}$ from which it will follow that $\operatorname{Im}\left\{F_{1}(z)\right\}$ changes sign exactly twice on $E_{1}$.

To this end let $t=\pi / 2$, then $u-\pi / 4=i \epsilon$. Substituting this value of $u-\pi / 4$ into (4.20) yields

$$
\operatorname{Im}\{F(u)\}=-\frac{1}{8} \frac{c n \epsilon}{s n^{3} \epsilon d n^{3} \epsilon}\left(k^{2} k^{\prime 2} s n^{4} \epsilon-d n^{4} \epsilon\right) .
$$

Since $0<k<1,0<k^{\prime}<1, k^{\prime}>2^{-\frac{1}{2}}$, sn $\epsilon \rightarrow 0, c n \epsilon \rightarrow 1, d n \epsilon \rightarrow 1$ with $\epsilon$ tending to zero, it follows that there is a $\delta_{1}>0$ such that $\operatorname{Im}\{F(u)\}>0$ for each $\epsilon$ satisfying the relation $0<\epsilon<\delta_{1}$, whenever $t=\pi / 2$.

Next let $t=3 \pi / 2$, then $u-\pi / 4=K+i \epsilon$ in which case

$$
\operatorname{Im}\{F(u)\}=\frac{1}{8} \frac{s n \epsilon d n \epsilon}{c n^{3} \epsilon}\left(k^{2}-k^{\prime 2} c n^{4} \epsilon\right) .
$$

Let $\eta>1 / \sqrt{2}$ be given, then for $k^{\prime}>\eta>2^{-\frac{1}{2}}$ there exists a $\delta_{2}>0$ such that $\operatorname{Im}\{F(u)\}<0$ for each $\epsilon$ with $0<\epsilon<\delta_{2}$ whenever $t=3 \pi / 2$. Hence there is a $\delta>0$ such that $\operatorname{Im}\left\{F_{1}(z)\right\}$ changes sign at least once on each ellipse $E_{\epsilon}$ confocal with $E$ situated in the boundary strip $0<\epsilon<\delta$ whenever $\pi / 2<t<$ $3 \pi / 2$. Since $F\left(\frac{1}{2} t-\pi / 4+i \epsilon+\pi\right)=F\left(\frac{1}{2} t-\pi / 4+i \epsilon\right)$ it follows that Im $\left\{F_{1}(z)\right\}$ changes sign at least twice on each ellipse $E_{\epsilon}$ in the boundary strip.

Upon differentiating $F^{\prime}(u)$ we find

$$
\begin{aligned}
& \frac{d}{d u}(F(u))=-\frac{1}{8}\left[\frac{2\left(1+k^{2}\right) \operatorname{sn}^{2}(u-\pi / 4+i \log R)-3}{s n^{4}(u-\pi / 4+i \log R)}\right. \\
&\left.\frac{2\left(1+k^{2}\right) s n^{2}(u-\pi / 4)-3}{\operatorname{sn}^{4}(u-\pi / 4)}\right]
\end{aligned}
$$

Employing the reduction formulae together with a lengthy computation reveals that

$$
\frac{d}{d t} \operatorname{Im}\{F(u)\}=-\frac{1}{16} \operatorname{snv} c n v d n v \operatorname{sn} \epsilon \operatorname{cn\epsilon } d n \epsilon g(x, \epsilon),
$$


where $v=\frac{1}{2} t-\pi / 4, x=\operatorname{snv}, g(x, \epsilon)$ is a continuous function of $x$, except for $x=\epsilon=0$ and such that

$$
\begin{aligned}
\lim _{\epsilon \rightarrow 0} g(x, \epsilon)=x^{-6} z^{-6}\left\{4 x ^ { 6 } k ^ { 2 } ( k ^ { 2 } - 1 ) \left[\left(2 k^{2}-k^{4}\right) x^{2}\right.\right. & \left.+\left(1-2 k^{2}\right)\right] \\
& \left.+6\left[\left(1+k^{2}\right) x^{2}-2\right]\left(1-k^{2} x^{2}\right)^{3}\right\}
\end{aligned}
$$

which is negative since $0<x^{2} \leq 1, k^{\prime}>2^{-\frac{1}{2}}$. Under these conditions there is a boundary strip given by $0<\epsilon<\delta_{3}$ in which $g(x, \epsilon)<0$ on each ellipse $E_{\epsilon}$ in the strip. On $E_{\epsilon} d / d t(\operatorname{Im}\{F(u)\})$ vanishes at $v=0, v=\pi / 2$ which is equivalent to $t=\pi / 2, t=3 \pi / 2$. Hence we conclude that there is a boundary strip $G$ such that $\operatorname{Im}\left\{F_{1}(z)\right\}$ changes sign exactly twice on each ellipse interior to $G$ and confocal with $E$, provided $k^{\prime}>2^{-\frac{1}{2}}$, that is $F_{1}(z)$ belongs to $R$.

5. Starlike functions. Let us now consider the class of functions which are regular in $E$ and map $E$ onto a domain $D$ starlike with respect to the origin. By starlikeness with respect to the origin we shall mean the arg $\{f(\cos (t-i s))\}$, $0<s<s_{0}$, monotonically increases as $t$ increases from 0 to $2 \pi$. From this condition it follows that there exists a real number $t_{1}$ such that the variation of $\arg \{f(\cos (t-i s))\}$ between $t_{1}$ and $t_{1}+\pi$ is exactly $\pi$. For since $|\Delta \arg f|$ between $t_{1}$ and $t_{1}+2 \pi$ is exactly $2 \pi$, suppose that $G(t)=\arg \{f(\cos (t+\pi-$ $i s))\}-\arg \{f(\cos (t-i s))\}$ and $G\left(t_{0}\right)=\alpha \leq \pi, g\left(t_{0}+\pi\right)=\beta \geq \pi, 0 \leq t_{0} \leq \pi$. Then since $G(t)$ is a continuous function of $t$ for fixed $s$, it follows that there exists a $t_{1}, t_{0} \leq t_{1} \leq t_{0}+\pi$ such that $G\left(t_{1}\right)=\pi$. Hence there exist real numbers $\mu$ and $\nu$ such that $g(z)=e^{i \mu} f(\cos (t-\nu-i s))$ takes on real values when $z$ is real and on the ellipse. Also $g(z)$ is starlike and $\arg \{g(z)\}$ increases monotonically with $t$. We see then that $\operatorname{Im}\{g(z)\}>0,0<t<\pi$, and $\operatorname{Im} g(z)<0, \pi<t<2 \pi$. We should note here that $\mu$ and $\nu$ are functions of $s$.

Let $f(z)=\sum_{n=1}^{\infty} a_{n} T_{n}(z), a_{0}=0$ and $f(0)=0$, be regular in $E$ and starlike with respect to the origin. Let $g(z)=\sum_{n=1}^{\infty} b_{n} T_{n}(\cos (t-v-i s))$ where $b_{n}=e^{i \mu} a_{n}$. As in Theorem 2.1 we have that

$$
\operatorname{Re}\{\varphi(z)\}=\operatorname{Re}\{-i \sin t g(z)\} \geq 0 .
$$

A short computation yields

$$
\begin{array}{r}
\operatorname{Re}\{\varphi(z)\}=\sum_{n=1}^{\infty}\left[\alpha_{n} \sin n(t-\nu) \sinh n s+\beta_{n} \cos n(t-\nu) \cosh n s\right] \sin t \\
=\sum_{n=1}^{\infty} A_{n} \sin n t \sin t-B_{n} \cos n t \sin t
\end{array}
$$

where $\alpha_{n}+i \beta_{n}=b_{n}$ and $A_{n}=\alpha_{n} \cos n \nu \sinh n s+\beta_{n} \sin n \nu \cosh n s, B_{n}=\alpha_{n}$ $\sin n \nu \sinh n s-\beta_{n} \cos n \nu \cosh n s, n=1,2, \cdots$.

Rewriting (5.2) we have

$$
\operatorname{Re}\{\varphi(z)\}=\frac{1}{2}\left[A_{1}+\sum_{n=1}^{\infty}\left(A_{n+1}-A_{n-1}\right) \cos n t+\left(B_{n+1}-B_{n-1}\right) \sin n t .\right.
$$


Since by (5.1) the trigonometric series which is the right member of (5.3) is positive, we have as before

$$
\left|\left(A_{n+1}-A_{n-1}\right)-i\left(B_{n+1}-B_{n-1}\right)\right| \leq 2\left|A_{1}\right| \text {. }
$$

Now $A^{n}-i B_{n}=\frac{1}{2}\left(b_{n}^{*} R^{n}-b^{n} R^{-n}\right) e^{i n \nu}$ so that (5.4) becomes

$$
\begin{aligned}
\mid\left(b_{n+1}^{*} R^{n+1}-b_{n+1} R^{-(n+1)}\right) e^{i(n+1) \nu}-\left(b_{n-1}^{*} R^{n-1}-\right. & \left.b_{n-1} R^{-(n-1)}\right) e^{i(n-1) \nu} \mid \\
& \leq 2\left|b_{1}^{*} R-b_{1} R^{-1}\right| .
\end{aligned}
$$

In view of the fact that $A_{0}=B_{0}=0$ and employing induction we get

$$
\left|b_{n}^{*} R^{n}-b_{n} R^{-n}\right| \leq n\left|b_{1}^{*} R-b_{1} R^{-1}\right|
$$

or

$$
\left|b_{n}\right| \leq \frac{n}{R^{n}-R^{-n}}\left|b_{1}^{*} R-b_{1} R^{-1}\right|
$$

Returning to the original coefficients $a_{n}$ we have

$$
\begin{aligned}
\left|a_{n}\right| & \leq \frac{n}{R^{n}-R^{-n}}\left|e^{-i v} a_{1}^{*} R-e^{i v} a_{1} R^{-1}\right| \\
& \leq \frac{n\left|a_{1}\right|\left(R+R^{-1}\right)}{R^{n}-R^{-n}} .
\end{aligned}
$$

We state the results in the following

TheOREM 5.1. Let $f(z)=\sum_{n=1}^{\infty} a_{n} T_{n}(z), z \varepsilon E, a_{0}=0$ map $E$ onto a domain starlike with respect to the origin; then

$$
\left|a_{n}\right| \leq n\left|a_{1}\right| \frac{\left(R+R^{-1}\right)}{R^{n}-R^{-n}}, \quad n=1,2, \cdots .
$$

It is unlikely that (5.7) is sharp. The function (2.8) belongs to the class of starlike functions and more than likely plays the role of the extremal function in the above theorem.

6. The class D. Let $W=f(z)=\sum_{n=1}^{\infty} a_{n} T_{n}(z)$ be regular in $E$ and have one zero interior to $E$. Following Umezawa [7] we say that $f(z)$ has a diametral line if there exists a point $\zeta$ on $E$ such that the intersection of the straight line in the $W$ plane passing through the points $f(\zeta), 0, f(-\zeta)$ with the map of the ellipse is connected. If the direction of starlikeness of $f(z)$ is that of the diametral line we say $f(z)$ belongs to the class $D$. This implies that there exist real numbers $\mu$ and $\nu$ such that

$$
\begin{aligned}
& \operatorname{Im}\left\{f(z) e^{-i \mu}\right\} \geq 0 \text { for } \operatorname{Im}\left\{z \cos \nu+\left(1-z^{2}\right)^{\frac{1}{2}} \sin \nu\right\} \geq 0 \\
& \operatorname{Im}\left\{f(z) e^{-i \mu}\right\} \leq 0 \text { for } \operatorname{Im}\left\{z \cos \nu+\left(1-z^{2}\right)^{\frac{1}{2}} \sin \nu\right\} \leq 0
\end{aligned}
$$


for $z$ on $E$, where we note that $z \cos \nu+\left(1-z^{2}\right)^{\frac{1}{2}} \sin \nu=\cos (t-\nu-i s)$. Following a procedure analogous to that in $\$ 2$ we obtain a theorem similar to Theorem 5.1.

Theorem 6.1. Let $f(z)=\sum_{n=1}^{\infty} a_{n} T_{n}(z)$ belong to the class $D$; then

$$
\left|a_{n}\right| \leq \frac{n\left|a_{1} R-a_{1}^{*} R^{-1}\right|}{R^{n}-R^{-n}}, \quad n=1,2, \cdots \text {. }
$$

\section{ReFerences}

1. F. Bowman, Elliptic Functions, London, 1953.

2. N. G. De Bruisn, Ein Satz uber schlichte Funktionen, Nederlandse Akademie van Wetenschappen vol. 44(1941), pp. 47-49.

3. Zeev Nehari and Binyamin Schwarz, On the coefficients of univalent Laurent series, Proceedings of the American Mathematical Society vol. 5(1954), pp. 212-217.

4. M. S. Robertson, On the theory of univalent functions, Annals of Mathematics, vol. 37 (1936), pp. 374-408.

5. M. S. RoberTson, Analytic Functions star-like in one direction, American Journal of Mathematics, vol. 58(1936), pp. 465-472.

6. WeRner RogosinskI, Über positive harmonische Entwicklungen und typisch-reelle potenzreihen, Mathematische Zeitschrift, vol. 35(1932), pp. 93-121.

7. Toshio Umezawa, $A$ class of multivalent functions with assigned zeros, Proceedings of the American Mathematical Society, vol. 3(1952), pp. 813-820.

8. J. L. WALSH, Note on the orthogonality of Tchebycheff polynomials on confocal ellipses, Bulletin of the American Mathematical Society, vol. 40(1934), pp. 84-88.

9. E. T. Whittaker and G. N. Watson, Modern Analysis, Cambridge, 1952.

\section{UNIVERSITY OF KENTUCKY}

